\title{
Acute tumour response to the MEK1/2 inhibitor selumetinib (AZD6244, ARRY-142886) evaluated by non-invasive diffusion-weighted MRI
}

\author{
M Beloueche-Babari ${ }^{*}, 1$, Y Jamin ${ }^{1}$, V Arunan ${ }^{1}$, S Walker-Samuel ${ }^{1,4}$, M Revill ${ }^{2}$, P D Smith ${ }^{2}$, J Halliday ${ }^{2}$, \\ J C Waterton ${ }^{2}$, H Barjat ${ }^{2}$, P Workman ${ }^{3}, \mathrm{M} O$ Leach $^{*, 1}$ and S P Robinson ${ }^{1}$ \\ ${ }^{1}$ Cancer Research UK and EPSRC Cancer Imaging Centre, Division of Radiotherapy and Imaging, The Institute of Cancer Research \\ and The Royal Marsden NHS Foundation Trust, Sutton, Surrey SM2 5PT, UK; ${ }^{2}$ AstraZeneca, Alderley Park, Macclesfield, Cheshire \\ SK10 4TG, UK and ${ }^{3}$ Cancer Research UK Cancer Therapeutics Unit, Division of Cancer Therapeutics, The Institute of Cancer \\ Research, Sutton, Surrey SM2 5NG, UK
}

Background: Non-invasive imaging biomarkers underpin the development of molecularly targeted anti-cancer drugs. This study evaluates tumour apparent diffusion coefficient (ADC), measured by diffusion-weighted magnetic resonance imaging (DW-MRI), as a biomarker of response to the MEK1/2 inhibitor selumetinib (AZD6244, ARRY-142886) in human tumour xenografts.

Methods: Nude mice bearing human BRAF ${ }^{\mathrm{V} 600 \mathrm{D}}$ WM266.4 melanoma or BRAF $\mathrm{V}^{\mathrm{V} 600 \mathrm{E}}$ Colo205 colon carcinoma xenografts were treated for 4 days with vehicle or selumetinib. DW-MRI was performed before and $2 \mathrm{~h}$ after the last dose and excised tumours analysed for levels of phospho-ERK1/2, cleaved caspase 3 (CC3) and necrosis.

Results: Selumetinib treatment induced tumour stasis and reduced ERK1/2 phosphorylation in both WM266.4 and Colo205 tumour xenografts. Relative to day 0 , mean tumour ADC was unchanged in the control groups but was significantly increased by up to 1.6-fold in selumetinib-treated WM266.4 and Colo205 tumours. Histological analysis revealed a significant increase in necrosis in selumetinib-treated WM266.4 and Colo205 xenografts and CC3 staining in selumetinib-treated Colo205 tumours relative to controls.

Conclusion: Changes in ADC following treatment with the MEK1/2 inhibitor selumetinib in responsive human tumour xenografts were concomitant with induction of tumour cell death. ADC may provide a useful non-invasive pharmacodynamic biomarker for early clinical assessment of response to selumetinib and other MEK-ERK1/2 signalling-targeted therapies.

The RAS-BRAF-MEK-ERK1/2 pathway is an important signalling cascade in cancer that has attracted much attention as a key target for rational anti-cancer treatment. Deregulated ERK1/2 signalling drives many human cancers as a consequence of mutation or overexpression of proteins downstream in this oncogenic pathway.
Mutation of RAS oncogenes is very common in human cancer and $B R A F$ mutation, especially the V600E variant (with substitution of valine for glutamate) exhibiting very high kinase activity, is seen in $\sim 50 \%$ of malignant melanomas, $20 \%$ of colon carcinomas and $40 \%$ of thyroid cancers (Davies et al, 2002; Schubbert et al,

\footnotetext{
*Correspondence: Dr M Beloueche-Babari; E-mail: mouniab@icr.ac.uk or Professor MO Leach; E-mail: martin.leach@icr.ac.uk

${ }^{4}$ Present address: UCL Centre for Advanced Biomedical Imaging, Department of Medicine and Institute of Child Health, University College London, London WC1E 6DD, UK.
}

Received 25 April 2013; revised 17 July 2013; accepted 22 July 2013; published online 13 August 2013

(C) 2013 Cancer Research UK. All rights reserved 0007-0920/13 
2007; www.sanger.ac.uk/genetics/CGP/cosmic/). Thus, targeting MEK1/2/ERK1/2 signalling constitutes a key strategy for mechanismbased anti-cancer treatment with many inhibitors of BRAF-MEKERK1/2 signalling now in the clinic and in preclinical development. One promising agent is the MEK1/2 inhibitor selumetinib (AZD6244, ARRY-142886) that has recently undergone phase I/II clinical trials for the treatment of advanced melanoma and colorectal cancer (Adjei et al, 2008; Board et al, 2009; Bennouna et al, 2011) and completed a phase II combination study in non-small-cell lung cancer (Janne et al, 2012).

The development of molecularly targeted therapeutics such as BRAF and MEK1/2 inhibitors is assisted by the use of pharmacodynamic biomarkers to enable the monitoring of target inhibition and downstream pathway modulation and ultimately clinical response assessment and patient management (Banerji et al, 2008; Carden et al, 2009; Yap et al, 2010). Noninvasive functional imaging biomarkers are particularly advantageous, as they remove the need for surgical biopsy (and the associated issues of tumour heterogeneity and difficulty of access) and allow repeat measurements to be performed in the same patient and the same tumour as well as at multiple sites, thus permitting longitudinal assessment (Workman et al, 2006; Waterton and Pylkkanen, 2012). One imaging biomarker that is being evaluated in this context is the water proton apparent diffusion coefficient (ADC), derived from diffusion weightedmagnetic resonance imaging (DW-MRI), which provides image contrast based on the detection of the random (Brownian) thermally influenced motion of water molecules within tissues. With high sensitivity to motion over a few microns, DW-MRI can be applied to study the tumour microenvironment down to a cellular level. The distance travelled by a water molecule in a given time interval, the ADC, is inversely related to the presence of cell membrane boundaries, which indirectly informs on tissue cellularity (Padhani et al, 2009; Sinkus et al, 2012). DW-MRI has shown promise as a tool for diagnosis of cancer, monitoring its response to therapy in pre-clinical models and patients and predicting treatment outcome (Dzik-Jurasz et al, 2002; Koh et al, 2007; Kim et al, 2008; Padhani et al, 2009; Tunariu et al, 2012).

Treatment with BRAF-MEK-ERK1/2 signalling inhibitors, such as selumetinib, causes marked anti-tumour effects in many preclinical models. These drugs show higher potency in cancer cell lines harbouring oncogenic BRAF mutations but in vivo responses are also observed in tumour models that are $B R A F^{\mathrm{WT}}$, in particular those carrying RAS mutations (Solit et al, 2006; Davies et al, 2007; Ciuffreda et al, 2009; Yang et al, 2010).

The growth inhibitory action of the MEK1/2 inhibitor selumetinib has been associated with induction of tumour apoptosis in a range of pre-clinical models (Davies et al, 2007); we thus hypothesised that such effects could induce changes in tumour tissue cellular density that may be detectable by DW-MRI. Here we evaluate ADC as a non-invasive pharmacodynamic imaging biomarker for assessing the impact of MEK1/2 signalling inhibition with selumetinib in responsive human malignant melanoma and colon carcinoma xenograft models. We show that treatment with the MEK1/2 inhibitor selumetinib results in an increase in tumour ADC that is concomitant with induction of tumour cell death and that occurs in the absence of tumour volume changes.

\section{MATERIALS AND METHODS}

Materials. All tissue culture reagents were purchased from Gibco (Life Technologies Ltd, Paisley, UK). For animal anaesthesia, Hypnorm was purchased from Jansen Pharmaceuticals (High Wycombe, UK) and Hypnovel from Roche (Burgess Hill, UK). Selumetinib was obtained from AstraZeneca, Macclesfield, UK.
Cell culture. Human WM266.4 melanoma $\left(B R A F^{\mathrm{V} 600 \mathrm{D}}\right)$ and Colo205 $\left(B R A F^{\mathrm{V} 600 \mathrm{E}}\right)$ colorectal cancer cells were obtained from ATCC and grown as previously described (Beloueche-Babari et al, 2012). Cells were preserved and propagated according to ATCC's protocols, screened regularly for mycoplasma and passaged for no longer than 3 months.

Tumour xenograft models. Animals were treated in accordance with local and national ethical requirements and with the UK National Cancer Research Institute Guidelines for the Welfare and Use of Animals in Cancer Research (Workman et al, 2010). A total of $2-4 \times 10^{6}$ WM266.4 or Colo205 cells were injected subcutaneously into the flanks of 6-8 weeks-old female $\mathrm{NCr} / \mathrm{Nu}$ nude mice. Tumour volume was determined by calliper measurements of the tumour length $(l)$, width $(w)$, and depth $(d)$, and calculated using the formula: $(l \times w \times d) \times(\pi / 6)$.

When the tumours reached $500-550 \mathrm{~mm}^{3}$ in volume (3-4 weeks later), mice were randomised into two groups; a vehicle (water only) and a drug (selumetinib dissolved in water)-treated group. Mice bearing WM266.4 tumours (mean initial volume $559 \pm 51 \mathrm{~mm}^{3}$, $n=9)$ received $75 \mathrm{mg} \mathrm{kg}^{-1}$ selumetinib, while those with Colo205 tumours (mean initial volume $484 \pm 63 \mathrm{~mm}^{3}, n=6$ ) received $50 \mathrm{mg} \mathrm{kg}^{-1}$ selumetinib. These doses are similar to those previously used in mouse studies (Yeh et al, 2007) and were chosen on the basis of achieving suppression of ERK1/2 phosphorylation as well as induction of tumour stasis at the end of the treatment schedule. The vehicle-treated mice (mean initial tumour volumes of $592 \pm 46 \mathrm{~mm}^{3}(n=6)$ and $512 \pm 40 \mathrm{~mm}^{3}(n=5)$ for WM266.4 and Colo205, respectively) received water only. Drug and vehicle treatments were done by oral gavage twice daily, with each group receiving six doses in total over 4 days ( 1 dose late on day 0,2 doses on days 1 and 2 and 1 dose early on day 3 ).

Growth delay was monitored in a separate cohort of WM266.4 tumour-bearing mice, which were treated as described above ( $n=4$ for vehicle and $n=5$ for selumetinib), and tumour volume monitored for a further 7 days after the dosing had stopped.

MRI of tumours. Mice were anaesthetised with a single intraperitoneal injection of a Hypnovel-Hypnorm-water $(1: 1: 2)$ mixture at a dose of $10 \mathrm{ml} \mathrm{kg}^{-1}$. Animals were placed within a $3-\mathrm{cm}$ volume coil and positioned with the tumour at the isocentre of a Bruker 7 Tesla microimaging system (Bruker Instruments, Ettlingen, Germany). $\mathrm{T}_{2}$-weighted multi-slice anatomical images were initially acquired from 20 contiguous $1-\mathrm{mm}$ thick slices through the tumour to help identify the centre of the tumour (rapid acquisition with refocusing echoes RARE sequence, $\mathrm{TE}=36 \mathrm{~ms}$, $\mathrm{TR}=4500 \mathrm{~ms}, \quad 4$ averages, $128 \times 128$ phase encoding steps, $3 \times 3 \mathrm{~cm}^{2}$ field of view, RARE factor 8 ). DW-MRI was then performed on a single 1-mm thick axial slice through the largest extent of the tumour, using a diffusion-weighed spin-echo sequence $\left(\mathrm{TE}=32 \mathrm{~ms}, \mathrm{TR}=1500 \mathrm{~ms}, b\right.$-values $=150-750 \mathrm{~s} \mathrm{~mm}^{-2}$, five $b$-values, one average). Diffusion data were fitted using a novel Bayesian maximum a posteriori approach that takes the Rician noise distribution into account to calculate the ADC (WalkerSamuel et al, 2009). Also, $\mathrm{T}_{1}$ and $\mathrm{T}_{2}$ relaxation times were determined using an inversion recovery (IR) true-FISP sequence $(\mathrm{TI}=109-2902 \mathrm{~ms}, 25$ inversion times, $\mathrm{TR}=2.4 \mathrm{~ms}, \mathrm{TE}=1.2 \mathrm{~ms}$, eight averages, scan TR $=10 \mathrm{~s}$ ), and the data fitted using a similar $a$ posteriori approach but which also utilised the dual relaxation sensitivity of the IR true-FISP sequence (Walker-Samuel et al, 2009). Regions of interest outlining the tumour volume were drawn on high-resolution anatomical RARE images and used to derive the imaging parameters of interest. All data were fitted on a pixel-by-pixel basis using in-house software (ImageView), which provided maps of tumour spatial heterogeneity. The median value of each parameter in each tumour was determined.

MRI measurements were performed before (day 0 ) and $2 \mathrm{~h}$ following the last dose (on day 3 ). After the last scan, tumours were 
rapidly excised and divided into two parts; one frozen immediately at $-80{ }^{\circ} \mathrm{C}$ for western blotting and the other fixed in formal saline for histological analysis.

Assessment of MEK1/2 inhibition by western blotting. MEK1/2 signalling inhibition following selumetinib treatment in WM266.4 and Colo205 human tumour xenografts was assessed by western blotting for levels of phosphorylated ERK1/2 (P-ERK1/2). WM266.4 tumour differentiation was also assessed by monitoring the expression of the melanocyte lineage markers gp100 and Melan-A.

Tumour tissue homogenates were prepared as previously described and analysed by western blotting using standard protocols (Beloueche-Babari et al, 2012). The primary antibodies used were rabbit anti-P-ERK1/2, anti-total ERK1/2 (Cell Signaling Technology, Danvers, MA, USA), anti-gp100 and anti-Melan-A (Abcam, Cambridge, UK). Mouse anti-GAPDH antibody (ChemiCon, Billerica, MA, USA) was used to verify equal protein loading. The secondary anti-rabbit and anti-mouse antibodies were from GE Healthcare Life Sciences (Buckinghamshire, UK).

Immunohistochemistry. Following fixation in formal saline, tumour tissue was embedded in paraffin, and sections cut and stained with haematoxylin and eosin $(\mathrm{H} \& \mathrm{E})$ for the assessment of tumour necrosis or with antibodies against P-ERK1/2 or cleaved caspase-3 (CC3) (Cell Signaling Technology) using standard protocols as described previously (Davies et al, 2007). Semiquantitative scoring was carried out using image analysis. CC3 was scored using an algorithm developed for colour deconvolution (Aperio ScanScope, Aperio Technologies LTD, Vista, CA, USA) using thresholding. Colour thresholds were adjusted accordingly, and the data generated in both instances was a percentage of brown intensity score based on the region examined. The percentage of tumour necrosis was measured using Genie Aperio Technologies LTD pattern-recognition software for the automated quantitative assessment of viable tumour tissue and necrosis.

Statistical analysis. Significant changes in absolute tumour volume and quantitative MR parameters before and following treatment were identified using the Wilcoxon's matched pairs test. Relative changes in these parameters were expressed as a percentage of the pre-treatment value, and any significant differences between treated and control cohorts were identified using a paired two-tailed $t$-test. Changes in percentage of tumour necrosis and CC3 staining were assessed with the Mann-Whitney test. For all statistical tests, a
$P \leqslant 0.05$ was considered to be significant. Data are expressed as the mean \pm 1 s.e.m.

\section{RESULTS}

Selumetinib induces stasis in $\mathrm{BRAF}^{\mathrm{V} 600 \mathrm{D}} \mathrm{WM} 266.4$ melanomas concomitant with inhibition of MEK1/2-ERK1/2 signalling. Response of BRAF mutant tumours to MEK inhibitors is well established (Solit et al, 2006). As we were interested in early MRI indicators of response, we first confirmed the responsiveness to 4 days of selumetinib treatment in the $B R A F^{\mathrm{V} 600 \mathrm{D}}$ mutant WM266.4 human melanoma xenograft model. This short-term treatment resulted in significant growth inhibition. As shown in Figure 1A, the vehicle-treated WM266.4 tumours increased in volume to $146 \pm 8 \% \quad(P=0.001)$, while the volume of the drug-treated tumours remained unchanged $(103 \pm 7 \%, P=0.75)$, indicating tumour stasis. Tumours resumed growth after withdrawal of therapy, but the growth delay induced by selumetinib persisted for several days after the last dose (Figure 1B).

Confirming the expected effects on downstream signalling output, analysis of western blots from WM266.4 melanoma tissue showed a marked reduction in P-ERK1/2 levels in selumetinibtreated compared with vehicle-treated tumours (Figure 1C). Furthermore, MEK1/2 inhibition with selumetinib resulted in increased expression of the melanocyte lineage markers gp100 and Melan-A consistent with induction of melanoma cell differentiation following MEK1/2 signalling inhibition (Englaro et al, 1998; Kono et al, 2006).

Selumetinib increases ADC in WM266.4 human melanoma xenografts. Figure $2 \mathrm{~A}$ and $\mathrm{B}$ show anatomical $\mathrm{T}_{2}$-weighted images, parametric ADC maps and ADC distribution histograms acquired from a WM266.4 human melanoma xenograft before and following treatment with selumetinib, using the same dose regimen that inhibited MEK1/2 signalling and induced tumour stasis. Analysis of DW-MRI data indicated that tumour ADC increased in the drug-treated group to $159 \pm 21 \%$ relative to day $0(P=0.02)$, whereas ADC values remained unchanged in the vehicle-treated group $(116 \pm 13 \%, P=0.28)$. Quantification of these data is further summarised in Figure 2C.

Measurement of the native longitudinal relaxation time $T_{1}$ and the transverse relaxation time $\mathrm{T}_{2}$ in the selumetinib-treated tumours revealed no significant changes relative to day 0 values $(103 \pm 2 \%$ for $\mathrm{T} 1$ and $127 \pm 18 \%$ for $\mathrm{T} 2, P \geqslant 0.16)$.
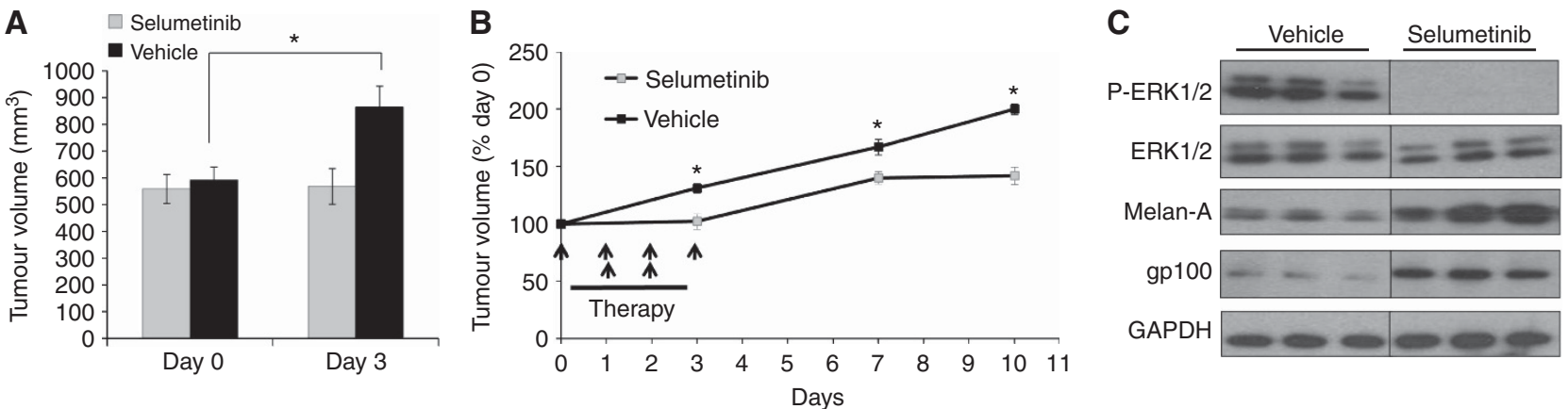

Figure 1. The anti-tumour effects of selumetinib therapy in V600E BRAF WM266.4 human melanoma xenografts. (A) Tumour volume measurements on days 0 and 3 showing the increase in tumour size in the vehicle-treated group over the course of treatment, while selumetinib (twice daily at $75 \mathrm{mg} \mathrm{kg}^{-1}$ ) induced tumour stasis. (B) The effect of selumetinib or vehicle treatment on WM266.4 tumour volume changes over the course of the therapy (dosing schedule indicated by black arrows) and 7 days after treatment withdrawal. (C) Western blots from representative tumours showing reduced ERK1/2 phosphorylation in selumetinib-treated compared with vehicle-treated WM266.4 human melanoma xenografts. An induction of WM266.4 melanoma tumour differentiation following selumetinib treatment is also observed, as indicated by the increased expression of the melanocyte markers gp100 and Melan-A. ${ }^{\star} P \leqslant 0.02$. 
A

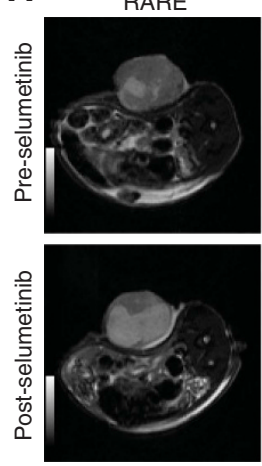

$\mathrm{ADC}$

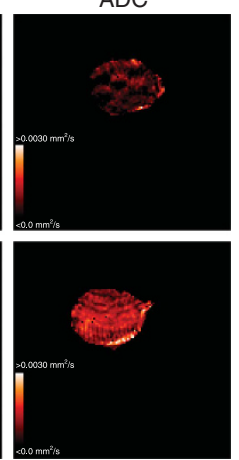

B

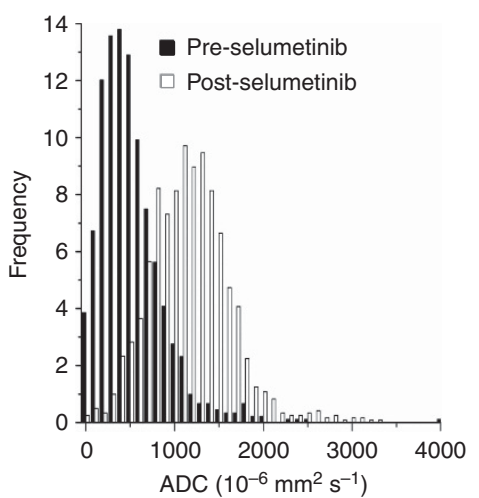

C

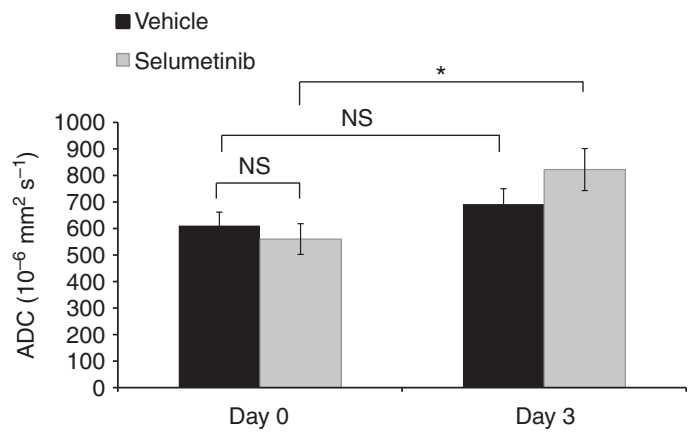

Figure 2. The effects of selumetinib therapy on ADC in WM266.4 human melanoma xenografts. (A) Representative anatomical $T_{2}$-weighted images and parametric ADC maps acquired before (top panel) and following treatment with the MEK1/2 inhibitor selumetinib (bottom panel) in WM266.4 tumours. (B) Frequency histograms showing the change in distribution of ADC values associated with the ADC maps shown in panel $\mathbf{A}$. (C) Summary of the changes in tumour ADC in the vehicle control and selumetinib cohorts before (day 0) and post treatment (day 3 ). Data are mean of median values, ${ }^{*} P=0.03$. NS: $P \geqslant 0.21$.

The selumetinib-induced rise in ADC is associated with increased tumour necrosis in WM266.4 melanoma xenografts. To investigate the biological basis for the increase in WM266.4 human melanoma ADC, we performed histological analyses on the excised tumour tissue samples. As shown in Figure $3 \mathrm{~A}$ and $\mathrm{B}$, $H \& E$ staining revealed an increase in the necrotic fraction in the selumetinib-treated cohort compared with the vehicle-treated tumours (mean scores of $58 \pm 10 \%$ vs $20 \pm 10 \%$, respectively; $P=0.03)$. CC3 staining showed no significant differences between selumetinib-treated and vehicle-treated WM266.4 tumours (Figure $3 \mathrm{~A}$ and $\mathrm{B}$ ), indicating no effect on tumour apoptosis. Staining for P-ERK1/2 (Figure 3A) showed reduced levels in the areas of the tumour that remained viable, thus further confirming the inhibitory effect of selumetinib on MEK-ERK1/2 signalling in WM266.4 tumours, in line with the western blot data.

These findings suggest that the increase in WM266.4 human melanoma tumour ADC is associated with increased tumour necrosis following selumetinib therapy.

Selumetinib induces an increase in ADC in BRAF $^{\mathrm{V} 600 \mathrm{E}}$ Colo205 colon tumour xenografts concomitant with inhibition of ERK1/2 signalling and tumour growth. To confirm that the changes in tumour ADC observed in the WM266.4 human melanoma xenograft after selumetinib treatment were not tumour type dependent, we investigated a second $B R A F$ mutant tumour model from a different tissue of origin, the $B R A F^{\mathrm{V} 600 \mathrm{E}}$ mutant Colo205 human colon carcinoma. Dosing with selumetinib caused tumour stasis in Colo205 xenografts with tumour volume remaining unchanged on day 3 compared with pre-treatment values $(101 \pm 4 \%, P=0.73)$. In contrast, and over the 4 days dosing schedule, the vehicle-treated Colo205 human colon carcinoma tumours grew to $135 \pm 6 \%$ compared with day 0 (Figures $4 \mathrm{~A}$, $P=0.002)$. Western blotting of excised tumour tissue on day 3 showed a marked suppression of ERK1/2 phosphorylation consistent with the inhibitory action of selumetinib on MEK1/2 (Figure 4B).

Analysis of DW-MRI data revealed a significant increase in mean tumour ADC following treatment with selumetinib on day 3, up to $147 \pm 18 \%$ of day 0 values $(P=0.01$; Figure $4 \mathrm{C})$. The vehicle-treated cohort showed no significant change in ADC on day 3 compared with day $0(102 \pm 19 \%, P=0.9)$.

The selumetinib-induced increase in ADC in Colo205 tumours is associated with induction of tumour apoptosis and necrosis. To qualify the changes in ADC observed in Colo205 xenografts,
A
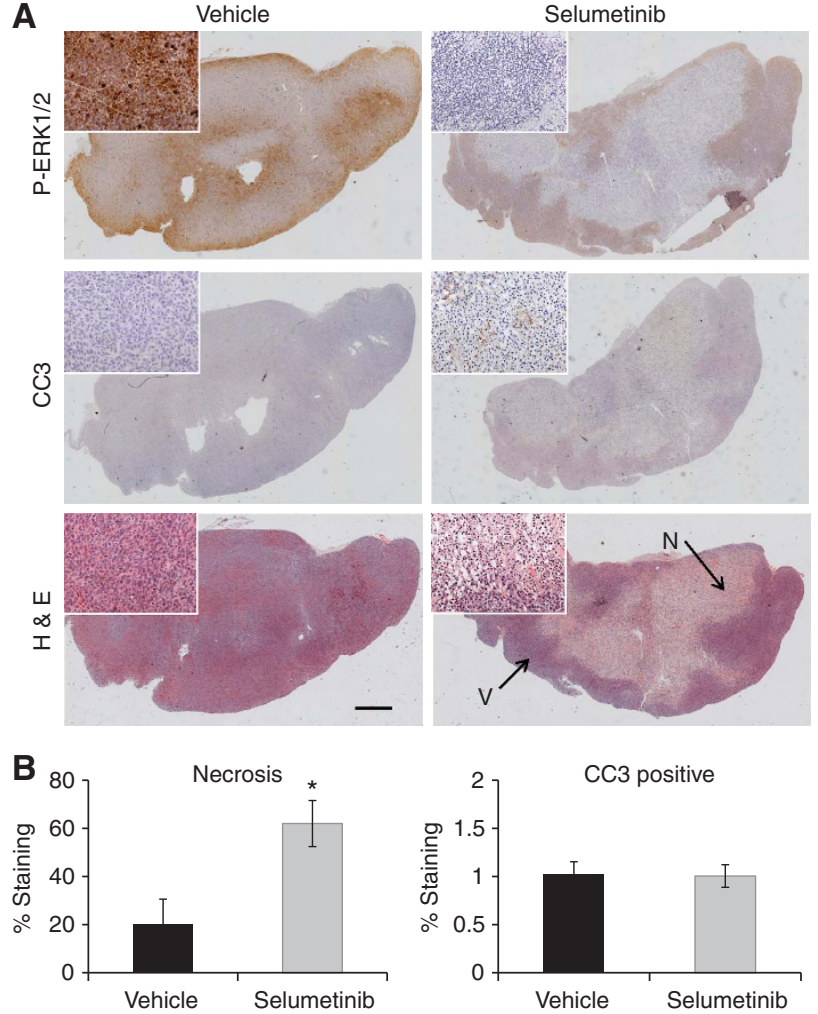

Figure 3. Ex vivo immunohistochemical assessment of the effect of selumetinib on WM266.4 human melanoma tumours. (A) Composite images of P-ERK1/2, CC3 and H\&E staining of vehicle (left) and selumetinib-treated (right) WM266.4 tumours. The insets represent higher magnification images. Arrows indicate the necrotic $(\mathrm{N})$ and viable $(V)$ tumour regions. (B) Summary of the H\&E staining analysis, demonstrating a statistically significant increase in the percentage of necrosis in the tumours treated with the MEK1/2 inhibitor selumetinib compared with the controls. ${ }^{*} P=0.05, \mathrm{Bar}=1 \mathrm{~mm}$.

we performed immunohistochemical assessment of the excised tumour tissue. As shown in Figure 5, P-ERK1/2 levels were markedly reduced in the selumetinib-treated tumours compared with the vehicle-treated controls consistent with our western 

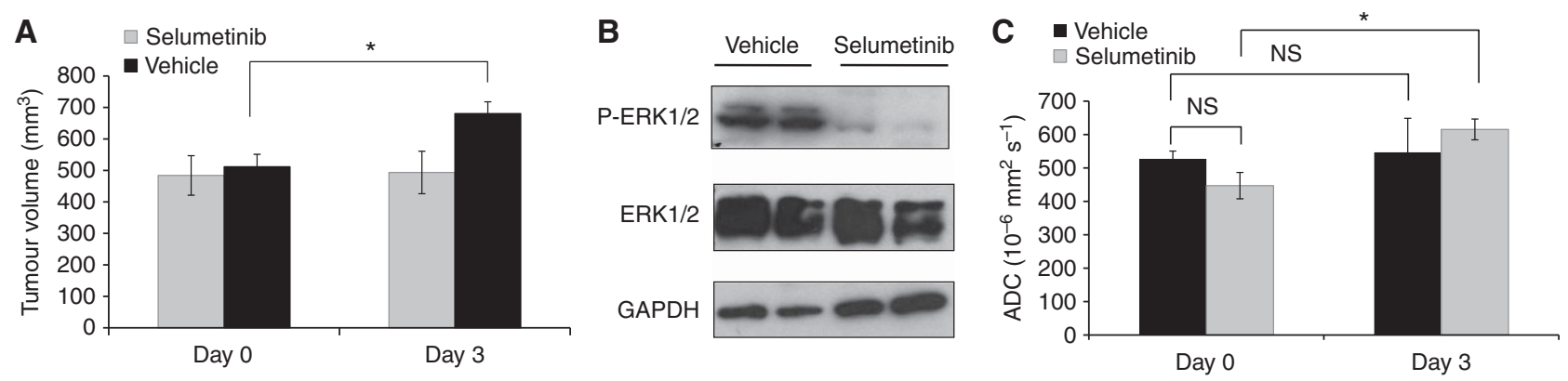

Figure 4. The effects of selumetinib on Colo205 human colon carcinoma growth, ERK1/2 signalling and tumour ADC. (A) Tumour volume measurements showing increased day 3/day 0 tumour size in the vehicle-treated group, while the selumetinib (p.o. twice daily at $50 \mathrm{mg} / \mathrm{kg}$ )treated tumours show stasis. (B) Western blots from representative tumours showing reduced ERK1/2 phosphorylation in selumetinib-treated compared with vehicle-treated Colo205 human tumour xenografts. (C) Summary of the DW-MRI data, showing no change in day $3 /$ day 0 tumour $A D C$ in the vehicle tumours, while ADC significantly increased in the selumetinib-treated group, $* P \leqslant 0.03$. NS: $P>0.14$.

blot analysis. Furthermore, we observed an increase in the tumour cell population staining positive for $\mathrm{CC} 3$ in the selumetinibtreated cohort $(18 \pm 2 \%)$ relative to the vehicle-treated tumours (10 $\pm 1 \%, P=0.017)$. H\&E staining showed that the percentage of tumour necrosis increased from $29 \pm 3 \%$ in the vehicle control tumours to $43 \pm 5 \%$ in the selumetinib-treated tumours $(P=0.03)$. These data indicate that the rise in Colo205 tumour ADC could result from increased tumour apoptosis and/or necrosis following selumetinib treatment. Overall, these results in Colo205 colon cancer xenografts were comparable to those seen in WM266.4 human melanoma xenografts.

\section{DISCUSSION}

The recent FDA approval of the BRAF inhibitor vemurafenib for the treatment of BRAF mutant metastatic melanoma (Flaherty et al, 2010), and the clinical activity reported with MEK1/2 inhibitors in the same disease (Flaherty et al, 2012), demonstrate the effectiveness of blocking the BRAF-MEK-ERK1/2 signalling pathway in BRAF-driven human melanoma tumours. The discovery and validation of pharmacodynamic, and preferably non-invasive, biomarkers that report on drug action is key for the rational development of therapies targeting the MEK1/2 pathway, as they allow target and pathway modulation to be monitored and therapeutic response to be assessed at an early stage and before any visible changes in tumour volume.

In the present study, we used DW-MRI to evaluate pharmacodynamic biomarkers of response to selumetinib, a MEK1/2selective inhibitor that has recently completed phase I/II testing in advanced melanoma and colorectal cancer (Adjei et al, 2008; Board et al, 2009; Bennouna et al, 2011) and completed a phase II combination study in non-small-cell lung cancer (Janne et al, 2012). This drug causes inhibition of growth and induction of apoptosis in a range of pre-clinical tumour models (Davies et al, 2007; Yeh et al, 2007). We therefore hypothesised that such effects could translate to reduced tumour cell density and increased extracellular space volume, leading to raised tumour ADC detectable by DW-MRI.

Two human tumour xenograft models were used to assess the effects of selumetinib on ADC: $B R A F^{\mathrm{V} 600 \mathrm{D}}$ WM266.4 melanoma and $B R A F^{\mathrm{V} 600 \mathrm{E}}$ Colo205 colorectal carcinoma. These animal models allow the relationship between imaging and pathological responses to be evaluated systematically, which is difficult to achieve in studies of cancer patients. Both of the tumour models harboured highly activating oncogenic BRAF mutations leading to dependency on MEK-ERK1/2 signalling for survival and increased sensitivity to MEK1/2 signalling inhibition (Solit et al, 2006; Davies et al, 2007). This BRAF mutation profile was chosen, as it is
A
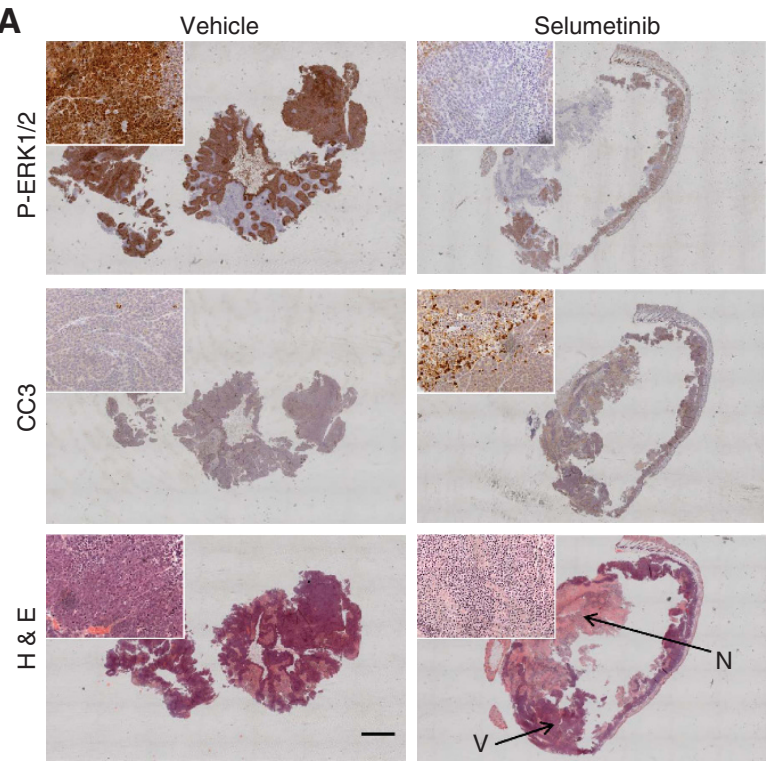

B
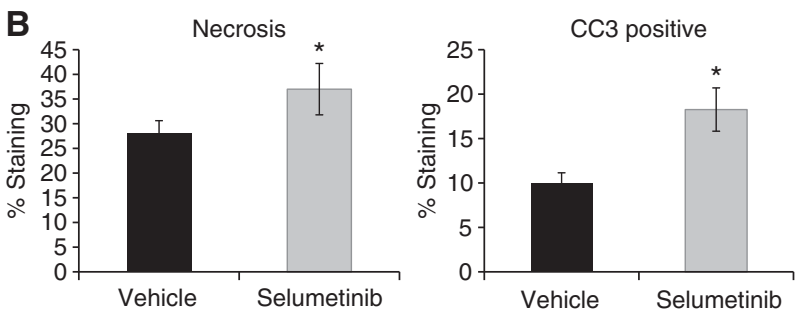

Figure 5. Histological assessment of apoptosis, necrosis and ERK1/2 activation in Colo205 human colon carcinoma xenografts following selumetinib therapy. (A) Composite images of Colo205 tumour tissue sections analysed by immunohistochemistry for P-ERK1/2, the apoptotic marker CC3 and H\&E. The insets represent higher magnification images. Arrows indicate the necrotic $(N)$ and viable $(V)$ tumour regions. (B) Quantitation of the percentage of tumour necrosis (H\&E staining) and the tumour cells staining positive for CC3 as determined by immunohistochemistry analysis in control and selumetinib-treated Colo205 human colon carcinoma tumours. ${ }^{*} P \leqslant 0.04, \mathrm{Bar}=1 \mathrm{~mm}$.

consistent with the genotype of the human tumours likely to be treated with BRAF-MEK1/2 signalling inhibitors in the clinic.

In $B R A F^{\mathrm{V} 600 \mathrm{D}} \mathrm{WM} 266.4$ human melanoma xenografts, selumetinib induced tumour growth inhibition concomitant with reduced ERK1/2 phosphorylation, thus confirming the inhibitory 
effects of the drug on MEK1/2. Furthermore, we also observed increased expression of melanocyte lineage markers consistent with induction of melanoma differentiation following MEK1/2 signalling inhibition (Englaro et al, 1998; Kono et al, 2006). DW-MRI analysis showed that selumetinib treatment led to a 1.6-fold rise in tumour ADC relative to pre-treatment values, whereas ADC was not changed in the control tumours. Increased tumour ADC suggests increased water diffusivity as a result of treatment-induced changes in tissue cellular packing density. Indeed analysis of excised tumour tissue by H\&E staining revealed an increase in the percentage of necrosis in the selumetinib-treated tumours relative to the vehicle-treated group. Thus, the rise in ADC in WM266.4 xenografts is likely to be caused by reduced restriction of water diffusion following induction of tumour necrosis and the associated loss of cellular membrane boundaries.

Similar effects were also observed in the BRAF ${ }^{\mathrm{V} 600 \mathrm{E}}$ Colo205 human colon carcinoma model, indicating that the ADC changes reported in WM266.4 tumours are not tumour model-specific.

An increase in percentage of tumour necrosis was observed in both Colo205 and WM266.4 xenografts. In the Colo205 tumour model, we also observed an increase in tumour apoptosis as revealed by CC3 staining, which was not seen in WM266.4 tumours. One possible explanation for this apparent difference could be the fact that caspase 3 cleavage is a dynamic process that has tumour model-dependent time course and amplitude, as previously reported (Davies et al, 2007). The increase in WM266.4 tumour necrosis could be the aftermath of a preceding induction in tumour apoptosis, although detailed time course assessment of CC3 levels would be required to test this hypothesis. These findings are consistent with a previous report associating increases in tumour ADC with induction of global cell death resulting from various mechanisms, rather than any one specific type (Morse et al, 2007).

Our findings, confirmed in two independent human tumour models, show that response to MEK1/2 inhibition with selumetinib results in elevated tumour ADC that occurred in the absence of changes in treated tumour volume. Hence ADC could have value as an early functional biomarker of response to drugs targeted at MEK1/2 signalling. In addition to restriction of tissue water diffusion, ADC can also be affected by blood flow and perfusion (Padhani et al, 2009). In our imaging protocol, the $b$-values used to calculate the ADC covered the range $150-750 \mathrm{~s} \mathrm{~mm}^{-2}$, which excludes contributions from tumour tissue perfusion to our ADC readouts (Padhani et al, 2009). Further work is warranted to assess the impact of selumetinib and other BRAF-MEK1/2-targeted therapies on tumour perfusion.

In addition to ADC measurements, we also evaluated native $T_{1}$ and $\mathrm{T}_{2}$ in WM266.4 tumours and observed no significant difference following selumetinib treatment. Native $T_{1}$ and $T_{2}$ are being actively investigated as putative pharmacodynamic biomarkers in oncology (McSheehy et al, 2010; Jamin et al, 2013). Our findings highlight the advantage of multiparametric MRI studies in assessing multiple imaging readouts in one experiment, thereby enabling the identification of the most robust biomarker of response, in this case tumour ADC.

Increases in tumour $\mathrm{ADC}$ have previously been observed in patients responding favourably to various forms of anti-cancer treatment, including radio- and chemotherapy (Hamstra et al, 2007; Koh et al, 2009). The use of DW-MRI to monitor response to agents targeted at oncogenic signalling has only been described in a small number of reports. An increase in tumour ADC was recorded clinically with favourable response to the tyrosine kinase inhibitor imatinib (Tang et al, 2011), the VEGFR inhibitor cediranib (Gerstner et al, 2010) and the RAF/VEGFR/PDGFR multikinase inhibitor sorafenib (Schraml et al, 2009). In preclinical cancer models, ADC increases were observed following treatment with the EGFR1 inhibitor gefitinib (Aliu et al, 2009), the Jak1/2 inhibitor AZD1480 (Loveless et al, 2012), the HIF inhibitors PX-478 and NSC-134754 (Jordan et al, 2005; Baker et al, 2012) and sorafenib (Karroum et al, 2013). These effects were associated with the induction of tumour cell death and the expected increase in extracellular space volume fraction and water diffusivity, in line with our findings.

DW-MRI is increasingly being incorporated into the clinical trials of novel agents aimed at signal transduction pathways (Tunariu et al, 2012), and findings from this study highlight the potential value of ADC measurement to inform on the anti-tumour effects of drugs targeting BRAF-MEK-ERK1/2 signalling. This would complement current work using 2-[ $\left.{ }^{18} \mathrm{~F}\right]$ fluoro-2-deoxyD-glucose positron emission tomography (FDG-PET) to noninvasively monitor response to BRAF-MEK1/2-targeted therapy in the clinic (Chapman et al, 2011). ADC measurements, especially when combined with multi-parametric MRI of the tumour microenvironment (e.g., vascularity), will provide valuable complementary information to FDG-PET, that is, combining evaluation of energetic metabolism with physiological assessment of perfusion and cellularity. The current study provides proof of concept for incorporating DW-MRI measurements in the clinical trials of BRAF-MEK1/2-targeted drugs and potentially other oncogenic signalling inhibitors expected to trigger tumour cell death.

A key advantage of imaging the intrinsic functional properties of tumours, such as cellular density, is the ability to longitudinally follow up drug-induced spatial as well as temporal changes in tumour biology over time, enabling detection of the onset of resistance in the clinic (Workman and Clarke, 2011; Al-Lazikani et al, 2012). This is particularly important for inhibitors of the MEK1/2 pathway where acquired drug resistance is, in many cases, mediated by parallel bypass signalling pathways involving RTK activation and where the assessment of P-ERK1/2 inhibition does not inform on the eventual tumour cell response to treatment (Nazarian et al, 2010; Corcoran et al, 2011). In the clinical setting, an increase in tumour ADC, providing an early non-invasive readout of downstream induction of cell death following MEK1/2ERK1/2 pathway inhibition in addicted cancers, would be more informative with regards to treatment outcome than molecular assessment of target modulation alone.

DW-MRI was able to longitudinally monitor the onset of chemotherapeutic drug resistance in an animal tumour model (Lee et al, 2006). It would therefore be of interest to evaluate ADC in tumours with varying degrees of sensitivity to BRAF-MEK1/2ERK1/2 signalling inhibitors, including those with intrinsic and acquired drug resistance. This will be key for objectively assessing the value and limitations of ADC as a potential pharmacodynamic biomarker of drug activity in pre-clinical models and optimise its use for clinical trials.

In summary, we have shown that MEK1/2 inhibition with selumetinib induces a rise in $\mathrm{ADC}$ values in responsive human melanoma and colon carcinoma xenografts that was concomitant with induction of cell death and that occurred in the absence of visible changes in tumour volume. Thus ADC could be useful as an early non-invasive biomarker of response to selumetinib and other inhibitors of MEK1/2 signalling in the clinic. DW-MRI can be readily incorporated into non-invasive imaging protocols forming part of clinical trials, thus allowing our findings to be translated to patient studies where the value of tumour ADC as a non-invasive biomarker of response to BRAF-MEK1/2-ERK1/2 signalling inhibitors can be assessed.

\section{ACKNOWLEDGEMENTS}

We thank the staff at the Biological Services Unit, ICR, for animal care and maintenance. This work was funded by the Cancer Research UK (grants C1060/A6916, C1060/A5117, C309/A8274 
and C107/A10433). In addition, we also acknowledge the support received from the Cancer Research UK and the EPSRC Cancer Imaging Centre in association with the MRC and Department of Health (England) grant C1060/A10334 and the NHS funding to the NIHR Biomedical Research Centre. SPR is the recipient of a Royal Society University Research Fellowship and research funding from AstraZeneca. PW is a Cancer Research UK Life Fellow. MOL is an NIHR Senior Investigator.

\section{CONFLICT OF INTEREST}

MR, PDS, JH, JCW and HB are or were employees of AstraZeneca, which has a commercial interest in MEK1/2 signalling inhibitors. $\mathrm{SPR}$ is a recipient of research funding from AstraZeneca. The other authors declare no conflict of interest.

\section{REFERENCES}

Adjei AA, Cohen RB, Franklin W, Morris C, Wilson D, Molina JR, Hanson LJ, Gore L, Chow L, Leong S, Maloney L, Gordon G, Simmons H, Marlow A, Litwiler K, Brown S, Poch G, Kane K, Haney J, Eckhardt SG (2008) Phase I pharmacokinetic and pharmacodynamic study of the oral, small-molecule mitogen-activated protein kinase kinase 1/2 inhibitor AZD6244 (ARRY-142886) in patients with advanced cancers. J Clin Oncol 26: 2139-2146.

Al-Lazikani B, Banerji U, Workman P (2012) Combinatorial drug therapy for cancer in the post-genomic era. Nat Biotechnol 30: 679-692.

Aliu SO, Wilmes LJ, Moasser MM, Hann BC, Li KL, Wang D, Hylton NM (2009) MRI methods for evaluating the effects of tyrosine kinase inhibitor administration used to enhance chemotherapy efficiency in a breast tumor xenograft model. J Magn Reson Imaging 29: 1071-1079.

Baker LC, Boult JK, Walker-Samuel S, Chung YL, Jamin Y, Ashcroft M, Robinson SP (2012) The HIF-pathway inhibitor NSC-134754 induces metabolic changes and anti-tumour activity while maintaining vascular function. Br J Cancer 106: 1638-1647.

Banerji U, de BJ, Judson I, Kaye S, Workman P (2008) Biomarkers in early clinical trials: the committed and the skeptics. Clin Cancer Res 14: 2512-2514.

Beloueche-Babari M, Arunan V, Troy H, te Poele RH, te Fong AC, Jackson LE, Payne GS, Griffiths JR, Judson IR, Workman P, Leach MO, Chung YL (2012) Histone deacetylase inhibition increases levels of choline kinase alpha and phosphocholine facilitating noninvasive imaging in human cancers. Cancer Res 72: 990-1000.

Bennouna J, Lang I, Valladares-Ayerbes M, Boer K, Adenis A, Escudero P, Kim TY, Pover GM, Morris CD, Douillard JY (2011) A Phase II, openlabel, randomised study to assess the efficacy and safety of the MEK1/2 inhibitor AZD6244 (ARRY-142886) versus capecitabine monotherapy in patients with colorectal cancer who have failed one or two prior chemotherapeutic regimens. Invest New Drugs 29: 1021-1028.

Board RE, Ellison G, Orr MC, Kemsley KR, McWalter G, Blockley LY, Dearden SP, Morris C, Ranson M, Cantarini MV, Dive C, Hughes A (2009) Detection of BRAF mutations in the tumour and serum of patients enrolled in the AZD6244 (ARRY-142886) advanced melanoma phase II study. Br J Cancer 101: 1724-1730.

Carden CP, Banerji U, Kaye SB, Workman P, de Bono JS (2009) From darkness to light with biomarkers in early clinical trials of cancer drugs. Clin Pharmacol Ther 85: 131-133.

Chapman PB, Hauschild A, Robert C, Haanen JB, Ascierto P, Larkin J, Dummer R, Garbe C, Testori A, Maio M, Hogg D, Lorigan P, Lebbe C, Jouary T, Schadendorf D, Ribas A, O’Day SJ, Sosman JA, Kirkwood JM, Eggermont AM, Dreno B, Nolop K, Li J, Nelson B, Hou J, Lee RJ, Flaherty KT, McArthur GA (2011) Improved survival with vemurafenib in melanoma with BRAF V600E mutation. N Engl J Med 364: 2507-2516.

Ciuffreda L, Del Bufalo D, Desideri M, Di Sanza C, Stoppacciaro A, Ricciardi MR, Chiaretti S, Tavolaro S, Benassi B, Bellacosa A, Foa R, Tafuri A, Cognetti F, Anichini A, Zupi G, Milella M (2009) Growth-inhibitory and antiangiogenic activity of the MEK inhibitor PD0325901 in malignant melanoma with or without BRAF mutations. Neoplasia 11: 720-731.
Corcoran RB, Settleman J, Engelman JA (2011) Potential therapeutic strategies to overcome acquired resistance to BRAF or MEK inhibitors in BRAF mutant cancers. Oncotarget 2: 336-346.

Davies BR, Logie A, McKay JS, Martin P, Steele S, Jenkins R, Cockerill M, Cartlidge S, Smith PD (2007) AZD6244 (ARRY-142886), a potent inhibitor of mitogen-activated protein kinase/extracellular signal-regulated kinase kinase $1 / 2$ kinases: mechanism of action in vivo, pharmacokinetic/ pharmacodynamic relationship, and potential for combination in preclinical models. Mol Cancer Ther 6: 2209-2219.

Davies H, Bignell GR, Cox C, Stephens P, Edkins S, Clegg S, Teague J, Woffendin H, Garnett MJ, Bottomley W, Davis N, Dicks N, Ewing R, Floyd Y, Gray K, Hall S, Hawes R, Hughes J, Kosmidou V, Menzies A, Mould C, Parker A, Stevens C, Watt S, Hooper S, Wilson R, Jayatilake H, Gusterson BA, Cooper C, Shipley J, Hargrave D, Pritchard-Jones K, Maitland N, Chenevix-Trench G, Riggins GJ, Bigner DD, Palmieri G, Cossu A, Flanagan A, Nicholson A, Ho JWC, Leung SY, Yuen ST, Weber BL, Siegler HF, Darrow TL, Paterson H, Marais R, Marshall CJ, Wooster R, Stratton MR, Futreal PA (2002) Mutations of the BRAF gene in human cancer. Nature 417: 949-954.

Dzik-Jurasz A, Domenig C, George M, Wolber J, Padhani A, Brown G, Doran S (2002) Diffusion MRI for prediction of response of rectal cancer to chemoradiation. Lancet 360: 307-308.

Englaro W, Bertolotto C, Busca R, Brunet A, Pages G, Ortonne JP, Ballotti R (1998) Inhibition of the mitogen-activated protein kinase pathway triggers B16 melanoma cell differentiation. J Biol Chem 273: 9966-9970.

Flaherty KT, Puzanov I, Kim KB, Ribas A, McArthur GA, Sosman JA, O'Dwyer PJ, Lee RJ, Grippo JF, Nolop K, Chapman PB (2010) Inhibition of mutated, activated BRAF in metastatic melanoma. $N$ Engl $J$ Med 363: 809-819.

Flaherty KT, Robert C, Hersey P, Nathan P, Garbe C, Milhem M, Demidov LV, Hassel JC, Rutkowski P, Mohr P, Dummer R, Trefzer U, Larkin JM, Utikal J, Dreno B, Nyakas M, Middleton MR, Becker JC, Casey M, Sherman LJ, Wu FS, Ouellet D, Martin AM, Patel K, Schadendorf D (2012) Improved survival with MEK inhibition in BRAF-mutated melanoma. N Engl J Med 367: 107-114

Gerstner ER, Chen PJ, Wen PY, Jain RK, Batchelor TT, Sorensen G (2010) Infiltrative patterns of glioblastoma spread detected via diffusion MRI after treatment with cediranib. Neuro-oncol 12: 466-472.

Hamstra DA, Rehemtulla A, Ross BD (2007) Diffusion magnetic resonance imaging: a biomarker for treatment response in oncology. J Clin Oncol 25: 4104-4109.

Jamin Y, Tucker ER, Poon E, Popov S, Vaughan L, Boult JK, Webber H, Hallsworth A, Baker LC, Jones C, Koh DM, Pearson AD, Chesler L, Robinson SP (2013) Evaluation of clinically translatable MR imaging biomarkers of therapeutic response in the TH-MYCN transgenic mouse model of neuroblastoma. Radiology 266: 130-140.

Janne PA, Tsang Shaw A, Rodrigues Pereira J, Jeannin G, Vansteenkiste J, Barrios CH, Franke FA, Grinsted L, Smith PD, Zazulina V, Smith IC, Crino L (2012) Phase II double-blind, randomized study of selumetinib (SEL) plus docetaxel (DOC) versus DOC plus placebo as second-line treatment for advanced KRAS mutant non-small cell lung cancer (NSCLC). J Clin Oncol 30(suppl): Abstract 7503.

Jordan BF, Runquist M, Raghunand N, Baker A, Williams R, Kirkpatrick L, Powis G, Gillies RJ (2005) Dynamic contrast-enhanced and diffusion MRI show rapid and dramatic changes in tumor microenvironment in response to inhibition of HIF-1alpha using PX-478. Neoplasia 7: $475-485$.

Karroum O, Mignion L, Kengen J, Karmani L, Leveque P, Danhier P, Magat J, Bol A, Labar D, Gregoire V, Bouzin C, Feron O, Gallez B, Jordan BF (2013) Multimodal imaging of tumor response to sorafenib combined with radiation therapy: comparison between diffusion-weighted MRI, choline spectroscopy and 18F-FLT PET imaging. Contrast Media Mol Imaging 8: $274-280$

Kim H, Morgan DE, Buchsbaum DJ, Zeng H, Grizzle WE, Warram JM, Stockard CR, McNally LR, Long JW, Sellers JC, Forero A, Zinn KR (2008) Early therapy evaluation of combined anti-death receptor 5 antibody and gemcitabine in orthotopic pancreatic tumor xenografts by diffusionweighted magnetic resonance imaging. Cancer Res 68: 8369-8376.

Koh DM, Blackledge M, Collins DJ, Padhani AR, Wallace T, Wilton B, Taylor NJ, Stirling JJ, Sinha R, Walicke P, Leach MO, Judson I, Nathan P (2009) Reproducibility and changes in the apparent diffusion coefficients of solid tumours treated with combretastatin A4 phosphate and bevacizumab in a two-centre phase I clinical trial. Eur Radiol 19: $2728-2738$. 
Koh DM, Scurr E, Collins D, Kanber B, Norman A, Leach MO, Husband JE (2007) Predicting response of colorectal hepatic metastasis: value of pretreatment apparent diffusion coefficients. Am J Roentgenol 188: 1001-1008.

Kono M, Dunn IS, Durda PJ, Butera D, Rose LB, Haggerty TJ, Benson EM, Kurnick JT (2006) Role of the mitogen-activated protein kinase signaling pathway in the regulation of human melanocytic antigen expression. Mol Cancer Res 4: 779-792.

Lee KC, Hall DE, Hoff BA, Moffat BA, Sharma S, Chenevert TL, Meyer CR, Leopold WR, Johnson TD, Mazurchuk RV, Rehemtulla A, Ross BD (2006) Dynamic imaging of emerging resistance during cancer therapy. Cancer Res 66: 4687-4692.

Loveless ME, Lawson D, Collins M, Nadella MV, Reimer C, Huszar D, Halliday J, Waterton JC, Gore JC, Yankeelov TE (2012) Comparisons of the efficacy of a Jak1/2 inhibitor (AZD1480) with a VEGF signaling inhibitor (cediranib) and sham treatments in mouse tumors using DCE-MRI, DW-MRI, and histology. Neoplasia 14: 54-64.

McSheehy PM, Weidensteiner C, Cannet C, Ferretti S, Laurent D, Ruetz S, Stumm M, Allegrini PR (2010) Quantified tumor t1 is a generic earlyresponse imaging biomarker for chemotherapy reflecting cell viability. Clin Cancer Res 16: 212-225.

Morse DL, Galons JP, Payne CM, Jennings DL, Day S, Xia G, Gillies RJ (2007) MRI-measured water mobility increases in response to chemotherapy via multiple cell-death mechanisms. NMR Biomed 20: 602-614.

Nazarian R, Shi H, Wang Q, Kong X, Koya RC, Lee H, Chen Z, Lee MK, Attar N, Sazegar H, Chodon T, Nelson SF, McArthur G, Sosman JA, Ribas A, Lo RS (2010) Melanomas acquire resistance to B-RAF(V600E) inhibition by RTK or N-RAS upregulation. Nature 468 : 973-977.

Padhani AR, Liu G, Koh DM, Chenevert TL, Thoeny HC, Takahara T, Dzik-Jurasz A, Ross BD, Van CM, Collins D, Hammoud DA, Rustin GJ, Taouli B, Choyke PL (2009) Diffusion-weighted magnetic resonance imaging as a cancer biomarker: consensus and recommendations. Neoplasia 11: 102-125.

Schraml C, Schwenzer NF, Martirosian P, Bitzer M, Lauer U, Claussen CD, Horger M (2009) Diffusion-weighted MRI of advanced hepatocellular carcinoma during sorafenib treatment: initial results. Am J Roentgenol 193: W301-W307.

Schubbert S, Shannon K, Bollag G (2007) Hyperactive Ras in developmental disorders and cancer. Nat Rev Cancer 7: 295-308.

Sinkus R, Van Beers BE, Vilgrain V, DeSouza N, Waterton JC (2012) Apparent diffusion coefficient from magnetic resonance imaging as a biomarker in oncology drug development. Eur J Cancer 48: 425-431.

Solit DB, Garraway LA, Pratilas CA, Sawai A, Getz G, Basso A, Ye Q, Lobo JM, She Y, Osman I, Golub TR, Sebolt-Leopold J, Sellers WR,
Rosen N (2006) BRAF mutation predicts sensitivity to MEK inhibition. Nature 439: 358-362.

Tang L, Zhang XP, Sun YS, Shen L, Li J, Qi LP, Cui Y (2011) Gastrointestinal stromal tumors treated with imatinib mesylate: apparent diffusion coefficient in the evaluation of therapy response in patients. Radiology 258: 729-738.

Tunariu N, Kaye SB, DeSouza NM (2012) Functional imaging: what evidence is there for its utility in clinical trials of targeted therapies? Br J Cancer 106: 619-628.

Walker-Samuel S, Orton M, McPhail LD, Robinson SP (2009) Robust estimation of the apparent diffusion coefficient (ADC) in heterogeneous solid tumors. Magn Reson Med 62: 420-429.

Waterton JC, Pylkkanen L (2012) Qualification of imaging biomarkers for oncology drug development. Eur J Cancer 48: 409-415.

Workman P, Aboagye EO, Balkwill F, Balmain A, Bruder G, Chaplin DJ, Double JA, Everitt J, Farningham DA, Glennie MJ, Kelland LR, Robinson V, Stratford IJ, Tozer GM, Watson S, Wedge SR, Eccles SA (2010) Guidelines for the welfare and use of animals in cancer research. Br J Cancer 102: 1555-1577.

Workman P, Aboagye EO, Chung YL, Griffiths JR, Hart R, Leach MO, Maxwell RJ, McSheehy PMJ, Price PM, Zweit J (2006) Minimally invasive pharmacokinetic and pharmacodynamic technologies in hypothesis-testing clinical trials of innovative therapies. J Natl Cancer Inst 98: $580-598$.

Workman P, Clarke PA (2011) Resisting targeted therapy: fifty ways to leave your EGFR. Cancer Cell 19: 437-440.

Yang H, Higgins B, Kolinsky K, Packman K, Go Z, Iyer R, Kolis S, Zhao S, Lee R, Grippo JF, Schostack K, Simcox ME, Heimbrook D, Bollag G, Su F (2010) RG7204 (PLX4032), a selective BRAFV600E inhibitor, displays potent antitumor activity in preclinical melanoma models. Cancer Res 70: 5518-5527.

Yap TA, Sandhu SK, Workman P, de Bono JS (2010) Envisioning the future of early anticancer drug development. Nat Rev Cancer 10: 514-523.

Yeh TC, Marsh V, Bernat BA, Ballard J, Colwell H, Evans RJ, Parry J, Smith D, Brandhuber BJ, Gross S, Marlow A, Hurley B, Lyssikatos J, Lee PA, Winkler JD, Koch K, Wallace E (2007) Biological characterization of ARRY-142886 (AZD6244), a potent, highly selective mitogenactivated protein kinase kinase 1/2 inhibitor. Clin Cancer Res 13: $1576-1583$.

This work is published under the standard license to publish agreement. After 12 months the work will become freely available and the license terms will switch to a Creative Commons AttributionNonCommercial-Share Alike 3.0 Unported License. 